\title{
Comparative validity of vitamin $C$ and carotenoids as indicators of fruit and vegetable intake: a systematic review and meta-analysis of randomised controlled trials
}

\author{
Mary Pennant ${ }^{1}$, Marinka Steur ${ }^{2,3}$, Carmel Moore ${ }^{2,3}$, Adam Butterworth ${ }^{2,3}$ and Laura Johnson ${ }^{2,3,4 *}$ \\ ${ }^{1}$ Public Health Department, Shire Hall, Cambridgeshire County Council, Cambridge CB3 OAP, UK \\ ${ }^{2}$ Cardiovascular Epidemiology Unit, Strangeways Research Laboratory, Department of Public Health and Primary Care, \\ University of Cambridge, Worts Causeway, Cambridge CB1 8RN, UK \\ ${ }^{3}$ Cambridge Institute of Public Health, University of Cambridge School for Clinical Medicine, Forvie Site, Cambridge \\ Biomedical Campus, Cambridge CB2 OSR, UK \\ ${ }^{4}$ Centre for Exercise Nutrition and Health Sciences, School for Policy Studies, University of Bristol, Bristol BS8 1TZ, UK
}

(Submitted 2 April 2015 - Final revision received 20 July 2015 - Accepted 22 July 2015 - First published online 9 September 2015)

\section{Abstract}

Circulating vitamin $\mathrm{C}$ and carotenoids are used as biomarkers of fruit and vegetable intake in research, but their comparative validity has never been meta-analysed. PubMed, EMBASE, CENTRAL, CINAHL and Web of Science were systematically searched up to December 2013 for randomised trials of different amounts of fruit and vegetable provision on changes in blood concentrations of carotenoids or vitamin C. Reporting followed PRISMA guidelines. Evidence quality was assessed using the GRADE system. Random effects meta-analysis combined estimates and meta-regression tested for sub-group differences. In all, nineteen fruit and vegetable trials ( $n$ 1382) measured at least one biomarker, of which nine ( $n$ 667) included five common carotenoids and vitamin C. Evidence quality was low and between-trial heterogeneity $\left(I^{2}\right)$ ranged from $74 \%$ for vitamin C to $94 \%$ for $\alpha$-carotene. Groups provided with more fruit and vegetables had increased blood concentrations of vitamin C, $\alpha$ carotene, $\beta$-carotene, $\beta$-cryptoxanthin and lutein but not lycopene. However, no clear dose-response effect was observed. Vitamin $\mathrm{C}$ showed the largest between-group difference in standardised mean change from the pre-intervention to the post-intervention period (sMD 0.94; $95 \%$ CI $0 \cdot 66,1 \cdot 22$ ), followed by lutein (SMD 0.70; $95 \%$ CI 0.37, 1.03) and $\alpha$-carotene (smD 0.63; $95 \%$ CI 0.25, 1.01), but all CI were overlapping, suggesting that none of the biomarkers responded more than the others. Therefore, until further evidence identifies a particular biomarker to be superior, group-level compliance to fruit and vegetable interventions can be indicated equally well by vitamin $\mathrm{C}$ or a range of carotenoids. High heterogeneity and a lack of dose-response suggest that individual-level biomarker responses to fruit and vegetables are highly variable.

Key words: Vitamin C: Carotenoids: Fruit: Vegetables: Biomarkers

Higher fruit and vegetable intake has been associated with reduced risk for CVD, all-cause mortality and specific types of cancer $^{(1-4)}$. The WHO has recommend $400 \mathrm{~g}$ of fruit or vegetable intake/ $\mathrm{d}^{(5)}$, equating to five $80 \mathrm{~g}$ portions, and encourages the evaluation of interventions to increase intake of fruits and vegetables $^{(5)}$. Adherence to advice in dietary interventions is frequently assessed by self-report tools ${ }^{(6)}$, which have known limitations ${ }^{(7-9)}$. Social approval bias specifically occurs in fruit and vegetable interventions, resulting in overestimated self-reported intakes $^{(9)}$. Objective measures of fruit and vegetable intake are, therefore, essential to improve confidence in research findings.

Blood-based biomarkers, resulting from the metabolism of fruits and vegetables in the body, have been proposed as objective indicators of fruit and vegetable intake ${ }^{(10)}$. Biomarkers correlate weakly with fruit and vegetable intake assessed by a range of self-report tools ${ }^{(11,12)}$ - for example, a meta-analysis estimated the correlation between dietary and plasma vitamin C to be just $r 0.3^{(13)}$. However, comparing biomarkers with self-reported intakes to establish validity is flawed because true intakes are poorly represented by self-report tools. Dietary randomised controlled trials (RCT), with direct observation or provision of different amounts of fruit and vegetables to different groups, provide a more robust way to validate biomarkers of changes in dietary intake. Randomisation may rule out confounding from other lifestyle factors, and the direct observation or provision of fruit and vegetables may allow true intakes to be more accurately estimated compared with self-reported intakes from groups randomised to different dietary advice (potential for differential priming for social desirability bias).

In a systematic review of RCT published up to April 2009(14), the most commonly measured and consistently responsive biomarkers for fruits and vegetables were carotenoids and

Abbreviations: RCT, randomised controlled trial; ROB, risk of bias; SMC, standardised mean change; SMD, standardised mean difference.

* Corresponding author: L. Johnson, fax +44 117 3310418, email Laura.Johnson@bristol.ac.uk 
vitamin C. However, there was no meta-analysis to quantify the responsiveness or examine the consistency of response of carotenoids and vitamin C. Furthermore, there was no comparative analysis of different biomarkers measured within the same set of studies, which would allow the relative validity of different biomarkers to be established. The present systematic review updates the existing review with a specific focus on the effect of changes in fruit and vegetable intake on blood concentrations of vitamin $\mathrm{C}$ and carotenoids in RCT with food intake directly observed or provided to participants. To provide a direct comparison of different biomarkers, our primary analysis focused on those trials in which a common set of vitamin $\mathrm{C}$ and five carotenoids was measured.

\section{Methods}

The review was reported according to items in the PRISMA statement (online Supplementary Table S1).

\section{Trial identification}

A previous systematic review provided studies carried out before 2009 for the present review ${ }^{(14)}$. Updated searches were conducted (by L. J.) from April 2009 (last search date of previous systematic review $^{(14)}$ ) to December 2013 in PubMed, EMBASE, CENTRAL, CINAHL and Web of Science using terms related to fruits and vegetables, dietary intervention studies and biomarkers (see online Supplementary information for detailed search strategy). Relevant systematic reviews were obtained and their reference lists were examined for additional references. Citations were screened by one reviewer (M. P. or L. J.) and hard copies of the relevant articles were obtained. These were screened by one reviewer (M. P.) and checked for inclusion by a second reviewer (L. J.).

\section{Inclusion and exclusion criteria}

RCT of different amounts of fruit and vegetable intake (where some food intake was observed or provided) with outcomes of plasma or serum vitamin $\mathrm{C}$ or carotenoids were included in the review. Interventions of any duration were considered for inclusion. Trials altering other aspects of the diet, in addition to fruit and vegetable intake - for example, low-fat diets - were excluded to avoid the possibility that changes in blood-based biomarkers may be a result of dietary changes other than fruit and vegetables. Intervention studies of a single fruit or vegetable were excluded. Findings from these types of interventions may underestimate the utility of biomarkers for measures of general fruit and vegetable intake as the single food source may contain a more limited range of nutrients. Trials where fruit and vegetable intake was encouraged through dietary advice were excluded as adherence to the advice is harder to estimate. Trials in healthy or unhealthy populations were included, including populations with high CVD risk factors or impaired glucose metabolism. However, trials in populations with abnormalities in micronutrient metabolism or vitamin-deficient populations were excluded. Trials were included if they reported biomarker measurements, either as changes from baseline or as baseline and post-intervention values, and if information was available on the amount of fruits and vegetables consumed in each intervention group.

\section{Data extraction}

Data on trial and population characteristics and outcomes were extracted into an Excel form that was piloted on a sample of trials before use (by M. P., M. S., L. J. and C. M.). Data extracted on trial characteristics included the type of trial (parallel or cross-over), duration of intervention, information on the duration of pre-intervention and within-intervention washout periods, the amount and types of fruits and vegetables consumed and the mode of administration (some meals eaten under supervision $v$. all meals at home), smoking status, fasting status at the time of biomarker measurement, the use of dietary supplements, inclusion and exclusion criteria and funding sources. Population characteristics included the sample size, country and type of sample - for example, clinical or general population - and participant demographics including age, sex and ethnicity were also extracted. Whenever available, data on baseline, post-treatment and change in biomarker concentrations were extracted for each trial arm. Where data on the amount of fruits and vegetables provided or biomarker levels were incomplete or lacked estimates of precision, authors were contacted. For four trials ${ }^{(15-18)}$, data were supplied by the authors and are included in the review.

\section{Quality assessment}

A risk of bias (ROB) assessment was conducted (by M. P.) using the Cochrane risk of bias tool ${ }^{(19)}$. Randomisation, allocation concealment, participant and assessor blinding, missing data and selective outcome reporting were assessed. Other items hypothesised to potentially introduce ROB were also included: the exclusion of participants taking supplements or smoking, participant fasting at the time of blood sampling, diet adherence monitoring and sufficient intervention washout periods (for cross-over trials) ( $\geq 4$ weeks). The ROB for each trial was considered on the basis of whether any of the items, individually or in combination with others, were likely to have introduced bias, and trials were assigned as having no, possible or high ROB. The overall quality of the evidence for each outcome was assessed using the GRADE system ${ }^{(20)}$ that considers (1) the ROB across trials contributing to that outcome, (2) heterogeneity in the meta-analysis, (3) directness or the generalisability of the population in the trial, (4) precision of the effect size and (5) risk of publication bias.

\section{Data analysis}

Standardised mean change (SMC) and SD of biomarker concentrations from the pre-intervention to the post-intervention period were computed using the baseline SD within each trial arm, owing to variation in the units reported across studies ( $\mu \mathrm{mol} / \mathrm{l} ; \mathrm{mg} / \mathrm{dl} ; \mu \mathrm{mol} / \mu \mathrm{mol}$ of cholesterol; $\mu \mathrm{mol} / \mathrm{mol}$ of lipid). Effect sizes (standardised mean difference (SMD) were the difference in the SMC of biomarkers between arms with higher $v$. lowest fruit and vegetable intake. The SE of the SMD was computed from the variance of the smc and the sample size in each arm. For trials with more than two arms, the arm with the lowest fruit and vegetable intake was compared against all the 
other arms. To account for the use of the lowest intake arm in multiple comparisons, the sample size of that arm was divided by the number of comparison groups within that study ${ }^{(21)}$. Fruit and vegetable intake was described in terms of number of portions using standard UK portion sizes - that is, one portion equates to $80 \mathrm{~g}$ of fruits or vegetables ${ }^{(22)}$.

Mean differences in changes in biomarkers between groups allocated different doses of fruits and vegetables across the whole study in cross-over designs were assumed to be the same as mean differences between groups in parallel study designs. Where average biomarker concentrations before intervention and after intervention were described using medians or geometric means, they were assumed to approximate the mean; $95 \%$ CI or interquartile ranges were transformed to approximate the SD assuming a normal distribution. Where data on change was not available, pre-intervention and postintervention mean (SD) concentrations were extracted, and mean change was computed by subtracting the pre-intervention mean from the post-intervention mean in each arm. The SD of the SMC was computed using standard equations ${ }^{(21)}$ based on the SD at baseline and the SD at follow-up within each arm and biomarker-specific correlations $(r)$ based on published associations between baseline and follow-up concentrations of biomarkers ${ }^{(23,24)}$. Post hoc sensitivity analyses were performed to check the influence of all assumptions on the results, and the pattern of findings was unaltered.

For each biomarker, SMD (SE) was pooled across all trials using random effects meta-analysis with inverse variance weights, and heterogeneity was estimated using $I^{2(25)}$. Heterogeneity was considered low or high if $I^{2}$ was $<25$ or $>75 \%$, respectively. For the primary analysis, data were combined for each biomarker for trials that included vitamin $\mathrm{C}$ and a common set of five carotenoids ( $\alpha$-carotene, $\beta$-carotene, $\beta$-cryptoxanthin, lutein and lycopene). Sub-group analyses planned a priori were conducted for each biomarker using meta-regression to investigate potential dose-response effect (difference in fruit and vegetable intake between arms in each trial in grams per d) and sources of heterogeneity, including differences by intervention duration (0-3 v. 4 + weeks, categories created based on data available); intervention compliance (meals observed $v$. eaten at home); trial design (cross-over $v$. parallel); health status (healthy $v$. unhealthy); location (Europe $v$. USA $v$. Asia-Pacific); type of food provided (fruits and vegetables $v$. vegetables only, categories created based on data available); baseline fruit and vegetable intake ( $<1 v .2-3 v$ v $4-5$ portions, categories created based on data available); fasting status (fasted $v$. not); blood sample fraction (plasma $v$. serum); ROB (low $v$. possible $v$. high); and sex (mixed $v$. male $v$. female). To check for a possible ceiling effect among participants with elevated biomarker concentrations, we also performed sub-group analyses using baseline biomarker concentrations (low $v$. high based on median split, categories created based on data available). For sub-group analyses, all trials with that biomarker measured were used, regardless of the simultaneous measurement of other biomarkers. As substantial $\left(I^{2}>75 \%\right)$ between-trial heterogeneity was observed, a post hoc sensitivity analysis was conducted to examine the effect of excluding trials with outlying results ( $>2$ SD from the SMD) from the analysis. Statistical evidence of association was considered important at $P<0 \cdot 05$. Data were analysed in Stata, version 12 (StataCorp LP).

\section{Results}

Trial selection

Of 3759 unique records, 144 full-text articles were assessed for inclusion, and nineteen trials were included in the review (Fig. 1). In all, nineteen trials were identified in this review, ten of which were also included in the previous systematic review $^{(14)}$. Out of the nineteen trials, nine ${ }^{(23,26-33)}$ assessed a common set of six biomarkers including five carotenoids and vitamin C (online Supplementary Table S2) and were included in the comparative (primary) analysis. Of the papers rejected on full-text screening, the majority were excluded on the basis of the intervention, often because trials involved only dietary advice or because the intervention targeted a single fruit or vegetable only. Other common reasons for exclusion were wrong study design (not RCT with food provision) or wrong outcomes (no biomarker concentrations).

\section{Trial characteristics}

Trial characteristics for all the included trials are shown in Table 1. Totally, twelve trials were conducted in healthy populations $^{(15,17,23,27,28,31,32,34-38)}$. Two trials were conducted in populations with increased CVD risk $^{(29,33)}$, and single trials were conducted in populations with obesity ${ }^{(39)}$, overweight ${ }^{(16)}$, hypertension ${ }^{(30)}$, elevated blood pressure $^{(18)}$ or chronic obstructive pulmonary disease ${ }^{(26)}$. Within-trial differences in intake of fruits and vegetables ranged from 2 to 13 portions/d. The sample sizes ranged from twenty to 246 participants (median 64). For the nine trials included in the comparative analysis, the difference in the amount of fruits and vegetables between arms ranged from 2 to 7 portions/d.

\section{Quality of the evidence}

In the GRADE assessment of the quality of each outcome in the meta-analysis, no outcomes were downgraded for imprecision or indirectness. However, most trials were considered to have some ROB (Fig. 2). Trials did not state that there was allocation concealment and patient blinding was not possible. In a number of studies, there were inadequate pre-intervention and within-intervention washout periods and uncertainties around the true ingested amounts of fruits and vegetables (less adherence monitoring) (Fig. 2). In the absence of washout periods, there was considered to be risk of pre-intervention or crosstreatment contamination. In trials where consumption of fruit and vegetables was not directly observed, there was considered to be a likely overestimation of the true ingested amount. A concern in some trials was the inclusion of participants using nutritional supplements, a lack of fasting at the time of outcome measurement and the inclusion of patients who smoked. Funnel plots suggested the possibility of publication bias and heterogeneity for $\alpha$-carotene, $\beta$-carotene, $\beta$-cryptoxanthin and vitamin $\mathrm{C}$ (based on the occurrence of studies outside of the 


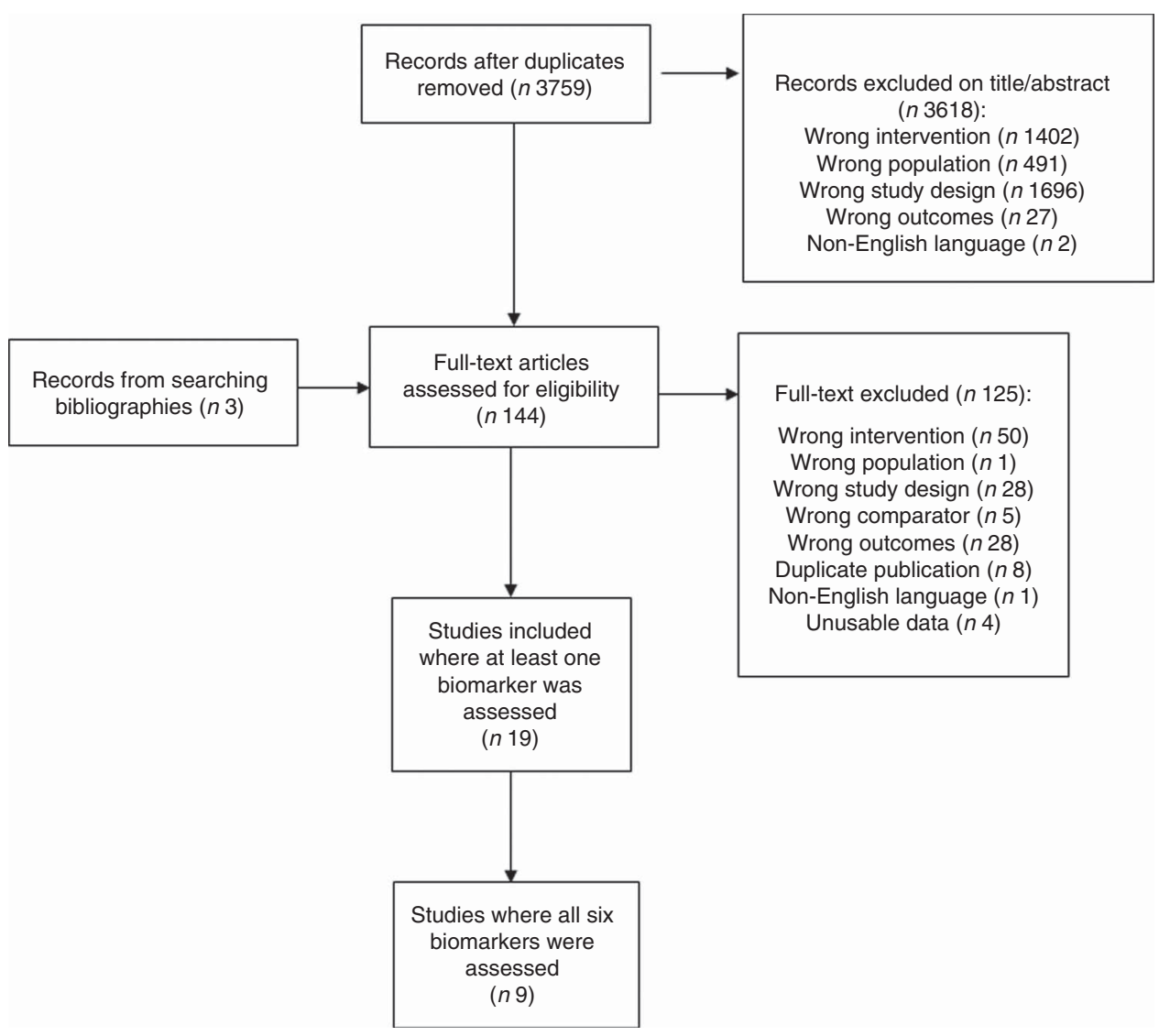

Fig. 1. PRISMA diagram of search results.

triangular region indicating where $95 \%$ of studies should be in the absence of bias or heterogeneity) (Fig. 3). All outcomes were downgraded for inconsistency as there was substantial heterogeneity in the meta-analysis. Overall, evidence for all outcomes was graded as having low quality.

\section{Findings}

The primary focus for this review was trials including measures of all six biomarkers so that their comparative utility could be assessed (Fig. 4). All biomarker concentrations, except lycopene, increased more from the pre-intervention to the postintervention period in the arm providing higher amounts of fruits and vegetables compared with the arm providing lower amounts: $\alpha$-carotene (SMD 0.63; $95 \%$ CI 0.25, 1.01), $\beta$-carotene (SMD 0.27; 95\%CI 0.08, 0.45), $\beta$-cryptoxanthin (SMD 0.52; $95 \% \mathrm{CI}$ $0.30,0.74$ ), lutein (SMD 0.70; $95 \%$ CI 0.37, 1.03) and vitamin C (SMD 0.94; $95 \%$ CI $0.66,1.22$ ). For lycopene, there was no evidence of greater change in plasma concentrations (SMD -0.02 ; $95 \% \mathrm{CI}-0 \cdot 27,0 \cdot 23)$ in response to higher fruit and vegetable intake. There was substantial between-trial heterogeneity in the pooled effects for all biomarkers $\left(I^{2}=74-94 \%\right)$. In the sensitivity analyses, where trials with extreme outlying results were excluded, seven out of nine trials remained in the analysis (online Supplementary Fig. S1). Effect sizes were smaller for all biomarkers but a similar pattern was observed, where there were significant effects for $\alpha$-carotene, $\beta$-carotene, $\beta$-cryptoxanthin, lutein and vitamin $\mathrm{C}$, but again no evidence of a difference for lycopene was observed. Heterogeneity was reduced for $\beta$-crytoxanthin, lutein, lycopene and vitamin C $\left(I^{2}=46-66 \%\right)$, but remained significant (online Supplementary Fig. S1). Further sensitivity analyses utilising information for each biomarker from all available studies (indirect comparisons) (online Supplementary Fig. S2) and excluding non-normally distributed data (online Supplementary Fig. S3) did not alter the pattern of the results.

Individual meta-analyses for each biomarker including up to nineteen trials are shown in the online Supplementary Fig. S4-S9. For these indirect comparisons, the same pattern was observed as for direct comparisons, with statistically significant effects for all biomarkers except lycopene. For these indirect analyses, we were able to additionally estimate the effects for zeaxanthin (online Supplementary Fig. S10) and total carotenoids (online Supplementary Fig. S11), which were available in a smaller number of studies. Both showed increases in response to high compared with low amounts of fruits and vegetables but were also highly heterogeneous $\left(I^{2}=84\right.$ and $93 \%$, respectively).

All trials providing data on at least one biomarker were included in the investigation of dose-response and sub-group analyses. In meta-regressions of within-trial difference in the amount of fruits and vegetables (grams/d) against SMD of biomarker level, there was no evidence of a dose-response effect (all $P>0.05$ ). When the difference in the amount of fruits and vegetables consumed in each arm was categorised into portions 
Table 1. Characteristics of nineteen randomised controlled trials of fruit and vegetable (F\&V) intake on biomarker concentrations

\begin{tabular}{|c|c|c|c|c|c|c|c|c|c|c|c|c|c|c|c|}
\hline \multirow[b]{2}{*}{ Author } & \multirow[b]{2}{*}{ Year } & \multirow[b]{2}{*}{ Population } & \multirow[b]{2}{*}{ Location } & \multirow[b]{2}{*}{ Design } & \multirow[b]{2}{*}{$n$} & \multirow{2}{*}{$\begin{array}{c}\text { Mean age } \\
\text { (years) }\end{array}$} & \multirow[b]{2}{*}{ Method } & \multirow[b]{2}{*}{ Intervention } & \multirow{2}{*}{$\begin{array}{l}\text { Intervention duration } \\
\text { (weeks) }\end{array}$} & \multirow{2}{*}{$\begin{array}{c}\text { Run-in } \\
\text { (weeks) }\end{array}$} & \multicolumn{2}{|c|}{$\begin{array}{l}\text { F\&V intake } \\
\text { (portions/d) }\end{array}$} & \multirow{2}{*}{$\begin{array}{l}\text { Blood } \\
\text { fraction }\end{array}$} & \multirow[b]{2}{*}{ Fasted state } & \multirow{2}{*}{$\begin{array}{l}\text { Smokers } \\
\text { excluded? }\end{array}$} \\
\hline & & & & & & & & & & & Baseline & Treatment & & & \\
\hline Baldrick et al. ${ }^{(26)}$ & 2012 & COPD & UK & Parallel & 81 & 62 & Provided F\&V, delivered to homes & $F \& V$ & 13 & 0 & $\begin{array}{l}\mathrm{GP}_{1} 1.4 \\
\mathrm{GP}_{2} 1.5\end{array}$ & $\begin{array}{l}\mathrm{GP}_{1} 1.9 \\
\mathrm{GP}_{2} 6 \cdot 1\end{array}$ & Plasma & Non-fasting & No \\
\hline Berry et al. ${ }^{(18)}$ & 2010 & Elevated blood pressure & UK & Cross-over & 57 & 45 & Provided F\&V, delivered to homes & $F \& V$ & 6 & 3 & $\begin{array}{l}\mathrm{GP}_{1} 3 \cdot 6 \\
\mathrm{GP}_{2} 3 \cdot 6 \\
\mathrm{GP}_{3} 3 \cdot 6\end{array}$ & $\begin{array}{l}\mathrm{GP}_{1} 3.6 \\
\mathrm{GP}_{2} 6.7 \\
\mathrm{GP}_{3} 8.0\end{array}$ & Plasma & Fasted & Yes \\
\hline Brevik et al. ${ }^{(34)}$ & 2004 & Healthy -students & Norway & Cross-over & 39 & 23 & Foods supplied and eaten under supervision & $F \& V$ & 2 & 1 & $\begin{array}{l}\mathrm{GP}_{1} \mathrm{NR} \\
\mathrm{GP}_{2} \mathrm{NR}\end{array}$ & $\begin{array}{l}\mathrm{GP}_{1} 3 \cdot 8 \\
\mathrm{GP}_{2} 9 \cdot 4\end{array}$ & Plasma & Fasted & Yes \\
\hline Briviba et al. ${ }^{(27)}$ & 2008 & Healthy - general & Germany & Parallel & 63 & NR & $\begin{array}{l}\text { Foods supplied and lunch eaten } \\
\text { under supervision }\end{array}$ & $F \& V$ & 3 & 1 & $\begin{array}{l}\mathrm{GP}_{1} 2 \cdot 8 \\
\mathrm{GP}_{2} 3 \cdot 3 \\
\mathrm{GP}_{3} 3 \cdot 1\end{array}$ & $\begin{array}{l}\mathrm{GP}_{1} 2.5 \\
\mathrm{GP}_{2} 5.8 \\
\mathrm{GP}_{3} 9.8\end{array}$ & Plasma & NR & Yes \\
\hline Broekmans et al. ${ }^{(28)}$ & 2000 & Healthy - low F\&V & The Netherlands & Parallel & 48 & 49 & $\begin{array}{l}\text { Foods supplied and dinner eaten } \\
\text { under supervision }\end{array}$ & $F \& V$ & 4 & NR & $\begin{array}{l}\mathrm{GP}_{1} 2 \cdot 0 \\
\mathrm{GP}_{2} 2 \cdot 0\end{array}$ & $\begin{array}{l}\mathrm{GP}_{1} 1 \cdot 3 \\
\mathrm{GP}_{2} 6 \cdot 3\end{array}$ & Plasma & Fasted & No \\
\hline Chong et al. (29) & 2013 & Increased CVD risk & UK & Parallel & 221 & 51 & Provided F\&V, delivered to homes & $F \& V$ & 18 & 2 & $\begin{array}{l}\mathrm{GP}_{1} 3 \cdot 9 \\
\mathrm{GP}_{2} 3 \cdot 8 \\
\mathrm{GP}_{3} 3 \cdot 4\end{array}$ & $\begin{array}{l}\mathrm{GP}_{1} 4.5 \\
\mathrm{GP}_{2} 7.6 \\
\mathrm{GP}_{3} 8 \cdot 1\end{array}$ & Plasma & Fasted & No \\
\hline Crane et al..$^{(16)}$ & 2011 & $\begin{array}{l}\text { Overweight (BMI 25-45 } \\
\mathrm{kg} / \mathrm{m}^{2} \text { ), post-menopausal } \\
\text { women }\end{array}$ & USA & Cross-over & 50 & 59 & Provided F\&V, delivered to homes & Vegetables only & 3 & 4 & $\begin{array}{l}\mathrm{GP}_{1} \mathrm{NR} \\
\mathrm{GP}_{2} \mathrm{NR} \\
\mathrm{GP}_{3} \mathrm{NR}\end{array}$ & $\begin{array}{l}\mathrm{GP}_{1} 1.6 \\
\mathrm{GP}_{2} 3.6 \\
\mathrm{GP}_{3} 7.7\end{array}$ & Plasma & Fasted & No \\
\hline Dragsted et al. ${ }^{(17)}$ & 2004 & Healthy - general & Denmark & Parallel & 48 & 26 & $\begin{array}{l}\text { Foods supplied and lunch eaten } \\
\text { under supervision }\end{array}$ & $F \& V$ & 4 & 0.4 & $\begin{array}{l}\mathrm{GP}_{1} 3 \cdot 3 \\
\mathrm{GP}_{2} 4 \cdot 1\end{array}$ & $\begin{array}{l}\mathrm{GP}_{1} 3.3 \\
\mathrm{GP}_{2} 7.5\end{array}$ & Plasma & Fasted & Yes \\
\hline Gill et al.(23) & 2004 & Healthy volunteers & UK & Parallel & 20 & 26 & Foods provided (NR where consumed) & Vegetables only & 2 & 1 & $\begin{array}{l}\mathrm{GP}_{1} \mathrm{NR} \\
\mathrm{GP}_{2} \mathrm{NR}\end{array}$ & $\begin{array}{l}\mathrm{GP}_{1} 0.0 \\
\mathrm{GP}_{2} 1.4\end{array}$ & Plasma & Fasted & NR \\
\hline Howe et al. ${ }^{(39)}$ & 2009 & Obese & USA & Parallel & 37 & 33 & Food provided at breakfast and lunch & $F \& V$ & 13 & NR & $\begin{array}{l}\mathrm{GP}_{1} \mathrm{NR} \\
\mathrm{GP}_{2} \mathrm{NR}\end{array}$ & $\begin{array}{l}\mathrm{GP}_{1} 1.2 \\
\mathrm{GP}_{2} 2 \cdot 5\end{array}$ & Serum & Fasted & No \\
\hline Martini et al. ${ }^{(38)}$ & 1995 & Healthy & USA & Cross-over & 23 & 26 & Ate on site or picked up to eat at home & Vegetables only & 1 & 0.7 & $\begin{array}{l}\mathrm{GP}_{1} \mathrm{NR} \\
\mathrm{GP}_{2} \mathrm{NR} \\
\mathrm{GP}_{3} \mathrm{NR}\end{array}$ & $\begin{array}{l}\mathrm{GP}_{1} 0.0 \\
\mathrm{GP}_{2} 6.8 \\
\mathrm{GP}_{3} 8.6\end{array}$ & Plasma & Fasted & Yes \\
\hline McCall et al. ${ }^{(30)}$ & 2009 & Hypertension & UK & Parallel & 147 & 52 & Food delivered to home, weekly phone calls & $F \& V$ & 8 & 4 & $\begin{array}{l}\mathrm{GP}_{1} 0.9 \\
\mathrm{GP}_{2} 1 . \cdot 1 \\
\mathrm{GP}_{3} 1.1\end{array}$ & $\begin{array}{l}\mathrm{GP}_{1} 1 \cdot 1 \\
\mathrm{GP}_{2} 3.2 \\
\mathrm{GP}_{3} 5.6\end{array}$ & Serum & Fasted & No \\
\hline Moller et al. ${ }^{(35)}$ & 2003 & Healthy - general & Denmark & Parallel & 48 & 26 & $\begin{array}{l}\text { Foods supplied and lunch eaten } \\
\text { under supervision }\end{array}$ & $F \& V$ & 4 & 0.6 & $\begin{array}{l}\mathrm{GP}_{1} 3 \cdot 3 \\
\mathrm{GP}_{2} 4 \cdot 2\end{array}$ & $\begin{array}{l}\mathrm{GP}_{1} 0.0 \\
\mathrm{GP}_{2} 7.5\end{array}$ & Plasma & Fasted & Yes \\
\hline Neville et al.(31) & 2013 & Healthy, older adults & UK & Parallel & 83 & 71 & Advice and home deliveries of $F \& V$ & $F \& V$ & 16 & 0 & $\begin{array}{l}\mathrm{GP}_{1} 1.4 \\
\mathrm{GP}_{2} 1.4\end{array}$ & $\begin{array}{l}\mathrm{GP}_{1} 1.8 \\
\mathrm{GP}_{2} 6.0\end{array}$ & Plasma & Fasted & No \\
\hline Rantala et al. ${ }^{(15)}$ & 2002 & Healthy women & Finland & Cross-over & 37 & 43 & Ate on site or picked up to eat at home & $F \& V$ & 5 & 2 & $\begin{array}{l}\mathrm{GP}_{1} \mathrm{NR} \\
\mathrm{GP}_{2} \mathrm{NR}\end{array}$ & $\begin{array}{l}\mathrm{GP}_{1} 2 \cdot 9 \\
\mathrm{GP}_{2} 8 \cdot 3\end{array}$ & Plasma & Fasted & Yes \\
\hline Thompson et al. ${ }^{(36)}$ & 2005 & $\begin{array}{l}\text { Healthy - women's health } \\
\text { interest group }\end{array}$ & USA & Parallel & 246 & 48 & $\begin{array}{l}\text { Cookbook with daily menus and recipes and } \\
\text { one-third of meals supplied }\end{array}$ & $F \& V$ & 4 & 2 & $\begin{array}{l}\mathrm{GP}_{1} 4.5 \\
\mathrm{GP}_{2} 4.5\end{array}$ & $\begin{array}{c}\mathrm{GP}_{1} 5.4 \\
\mathrm{GP}_{2} 13.8\end{array}$ & Plasma & NR & Yes \\
\hline Thompson et al. ${ }^{(37)}$ & 2005 & Healthy - unclear source & USA & Parallel & 64 & 49 & Foods prescribed & $F \& V$ & 2 & 0 & $\begin{array}{l}\mathrm{GP}_{1} \mathrm{NR} \\
\mathrm{GP}_{2} \mathrm{NR}\end{array}$ & $\begin{array}{l}\mathrm{GP}_{1} 5.4 \\
\mathrm{GP}_{2} 18.2\end{array}$ & Plasma & Non-fasting & NR \\
\hline van Het Hof et al. (32) & 1999 & Healthy - general & The Netherlands & Parallel & 55 & 22 & $\begin{array}{l}\text { Foods supplied (90\% of energy intake) } \\
\text { and partially eaten under supervision }\end{array}$ & Vegetables only & 4 & NR & $\begin{array}{l}\mathrm{GP}_{1} \mathrm{NR} \\
\mathrm{GP}_{2} \mathrm{NR}\end{array}$ & $\begin{array}{l}\mathrm{GP}_{1} 1.6 \\
\mathrm{GP}_{2} 6.1\end{array}$ & Plasma & Fasted & Yes \\
\hline Wallace et al. ${ }^{(33)}$ & 2013 & High CVD risk & UK & Parallel & 105 & 56 & $\begin{array}{l}\text { Advice plus weekly home deliveries of } F \& V \\
\text { telephone call from researcher weekly }\end{array}$ & $F \& V$ & 12 & 4 & $\begin{array}{l}\mathrm{GP}_{1} 1.7 \\
\mathrm{GP}_{2} 1.7 \\
\mathrm{GP}_{3} 1.6\end{array}$ & $\begin{array}{l}\mathrm{GP}_{1} 1.8 \\
\mathrm{GP}_{2} 3.8 \\
\mathrm{GP}_{3} 7.1\end{array}$ & Plasma & Fasted & No \\
\hline
\end{tabular}

COPD, chronic obstructive pulmonary disease; GP, group; NR, not reported 


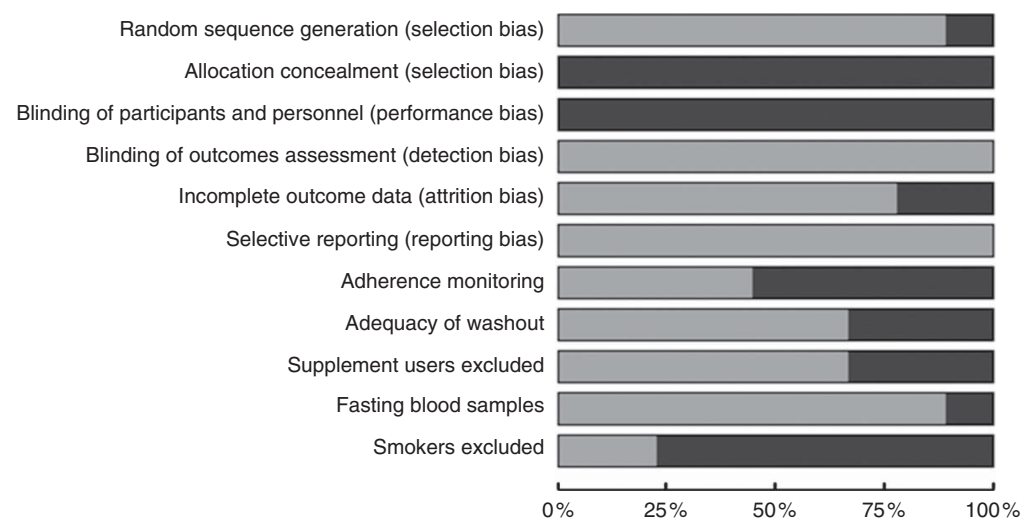

Fig. 2. Summary of risk of bias among the nine studies with six biomarkers measured.
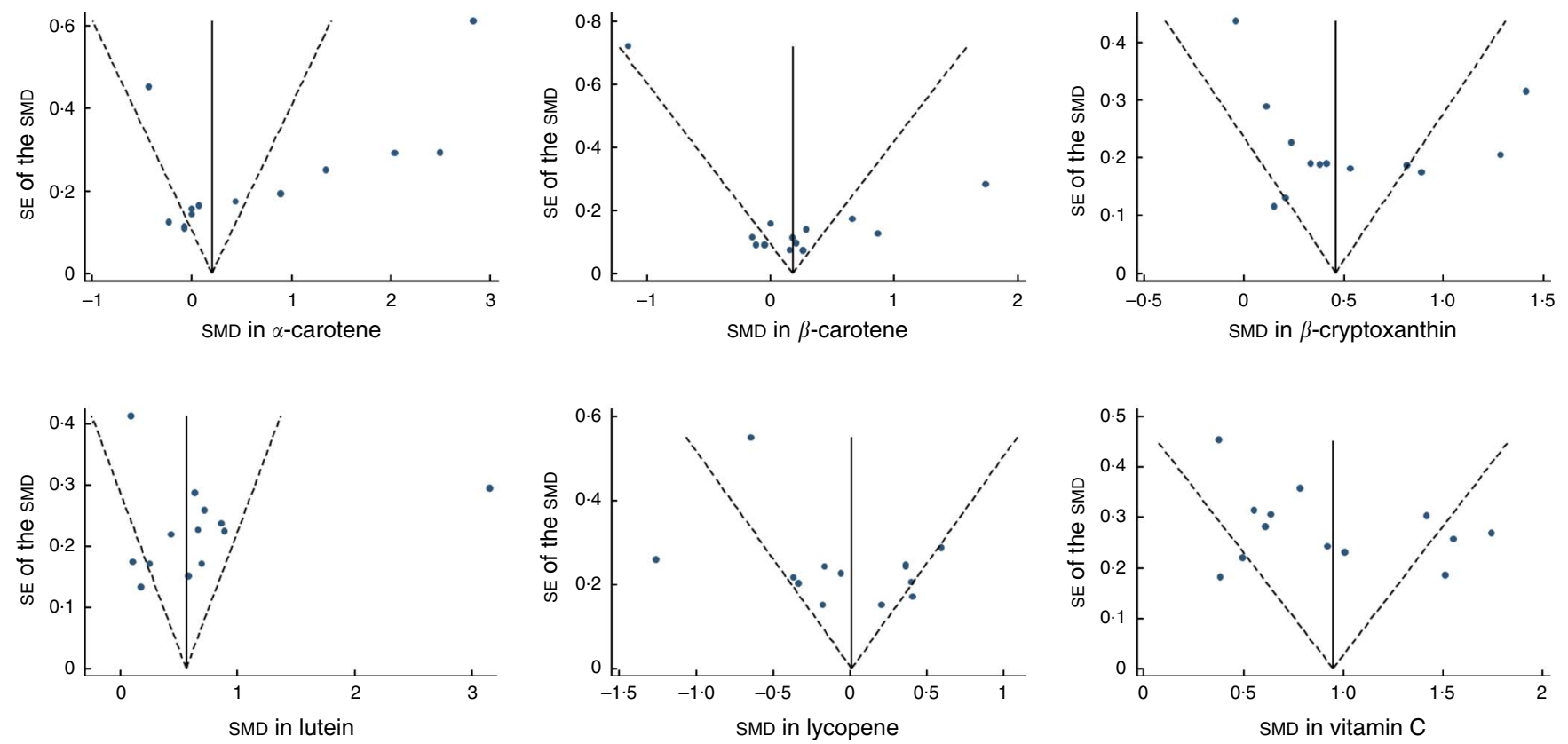

Fig. 3. Funnel plots of nine randomised controlled trials of different doses of fruit and vegetable intake on biomarker concentrations. SMD, standardised mean difference.

(2-3 v. 4-5 v. $>5$ portions), a trend towards higher biomarker concentrations among trials where the group difference in fruit and vegetable intake was greater emerged, but was only statistically significant for $\beta$-carotene ( $P=0 \cdot 01$, Fig. 5).

Other notable findings from sub-group analyses included stronger effects for $\alpha$-carotene, $\beta$-carotene, lutein and vitamin $\mathrm{C}$ in trials where participants ate meals under supervision compared with trials where food was eaten at home, accounting for $12-38 \%$ of the heterogeneity (online Supplementary Fig. S12). Shorter interventions (0-3 weeks) were associated with significantly greater effect sizes compared with longer ( $\geq 4$ weeks) interventions for $\alpha$ - and $\beta$-carotene. There were non-significant trends for a similar effect for lutein, lycopene and vitamin C, accounting for between 6 and $20 \%$ of the heterogeneity (online Supplementary Fig. S13). Trials in healthy populations tended to show greater effect sizes compared with trials in unhealthy populations (online Supplementary Fig. S14), and this was significant for $\alpha$ - and $\beta$-carotene (accounting for $17-18 \%$ of the heterogeneity). In the sensitivity analysis, excluding outlying results, there was still a significant effect of disease status for $\alpha$ - and $\beta$-carotene. In the sensitivity metaregressions including intervention delivery, duration and participant health status together, associations were unaltered (data not shown).

Trials conducted in the USA had significantly greater effect sizes compared with those conducted in Europe for $\alpha$ - and $\beta$-carotene (online Supplementary Fig. S15), which was robust to adjustment for other factors for $\alpha$-carotene. The effect size was greater for cross-over compared with parallel trials for $\beta$-carotene and lutein (online Supplementary Fig. S16), which 


\begin{tabular}{l|lcc}
\hline $\begin{array}{l}\text { D-Carotene } \\
\left(I^{2}=93.6 \% ; P<0.0001\right)\end{array}$ & 0.63 & $0.25,1.01$ \\
$\beta$-Carotene \\
$\left(I^{2}=88.1 \% ; P<0.0001\right)$
\end{tabular}

Fig. 4. Summary of pooled difference between arms consuming higher v. lower amounts of fruit and vegetables for standardised mean change (smc) of biomarkers from the pre-intervention to the post-intervention period in trials with all six biomarkers measured. SMC represents an SD of pre-intervention biomarker levels within each study. $P$ is an indicator of between-trial heterogeneity. Random effects meta-analysis was used to pool mean differences. Includes the following studies for ALL biomarkers: Baldrick et al. ${ }^{(26)}$, Briviba et al. ${ }^{(27)}$, Broekmans et al. ${ }^{(28)}$, Chong et al. ${ }^{(29)}$, Gill et al. ${ }^{(23)}$, McCall et al. ${ }^{(30)}$, Neville et al. ${ }^{(31)}$, van Het Hof et al. ${ }^{(32)}$, Wallace et $a l^{(33)}$. Total number of trials is nine; total number of arms being compared is twenty-two; total number of people included is 667.

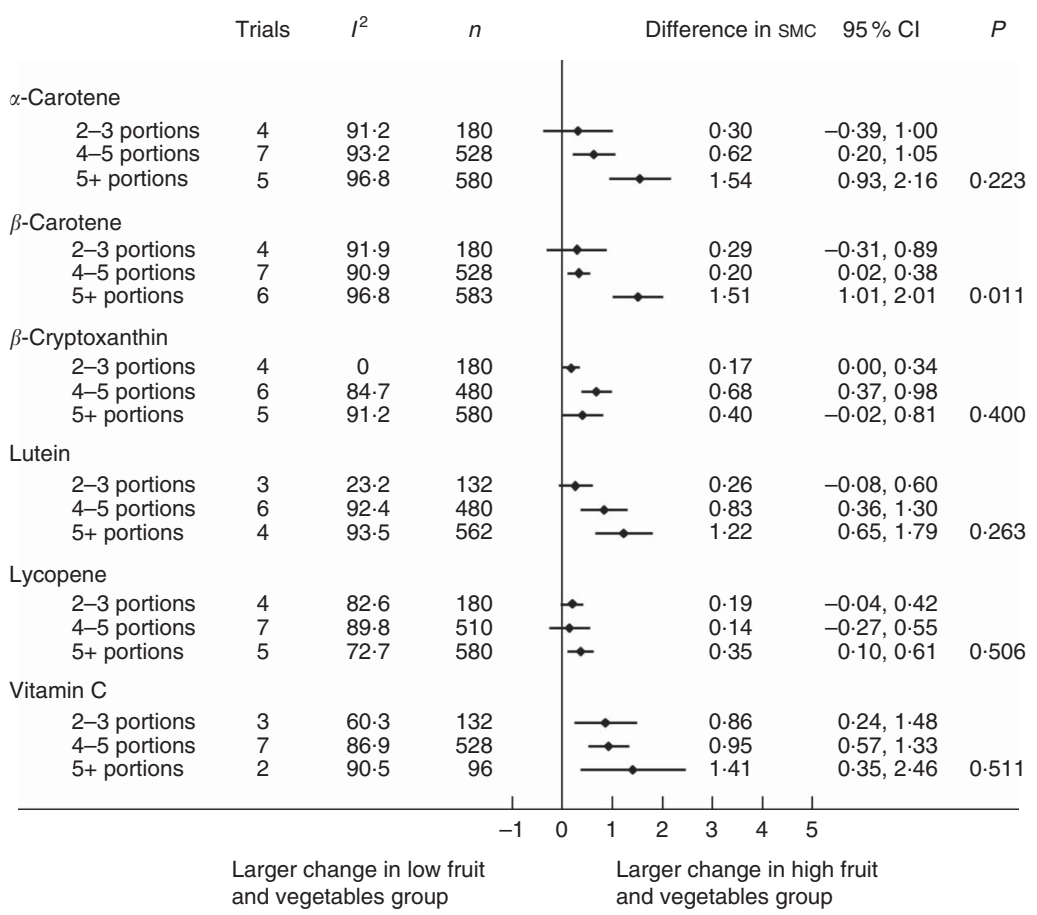

Fig. 5. Summary of pooled differences between arms consuming higher $v$. lower amounts of fruit and vegetables in standardised mean change (smc) of biomarkers from the pre-intervention to the post-intervention period in all trials with available data grouped by the amount of fruit and vegetables provided during the intervention. SMC represents an SD of pre-intervention biomarker levels within each study. $\mathcal{R}$ is an indicator of between-trial heterogeneity. Random effects meta-analysis was used to pool mean differences. $P$ value is from meta-regression test for trend across categories. Includes all studies up to $n 19$ based on availability of biomarker in each study.

was attenuated after adjustment for other factors (data not shown). For $\alpha$-carotene and lutein, there was a greater effect size for trials where vegetables alone were given compared with trials where both fruit and vegetables were given (online Supplementary Fig. S17), but these findings were not robust to adjustment (data not shown). There was no evidence of 
differences across sub-groups defined by baseline fruit and vegetable intake, fasting status, blood fraction (plasma or serum) or ROB (data not shown).

\section{Discussion}

In this systematic review, we identified nine additional RCT compared with a previous systematic review ${ }^{(14)}$, providing the largest evidence base to date for meta-analysis of the validity of carotenoids and vitamin $\mathrm{C}$ based on highly controlled validation studies. Although previous reviews have not been able to comment on the comparative validity of different biomarkers, our results highlight that vitamin $\mathrm{C}$ and four common carotenoids may all be equally useful as a biomarkers for objectively measuring general fruit and vegetable intake.

Similar to a previous systematic review ${ }^{(14)}$, vitamin $\mathrm{C}$ and carotenoids were identified as commonly used biomarkers for fruits and vegetables. In the previous systematic review, these biomarkers are qualitatively described as consistently responding to increased fruit and vegetable intakes. Our meta-analysis provides quantitative evidence to support that vitamin $\mathrm{C}, \alpha$ - and $\beta$-carotene, $\beta$-cryptoxanthin and lutein all increase in response to a high fruit and vegetable intake, but high heterogeneity estimates suggest a lack of consistency in the size of the response observed between studies.

Meta-regression of fruit and vegetable dose on changes in biomarker concentrations showed no evidence of a doseresponse relationship for any biomarkers. Although pooled biomarker responses in sub-groups defined by increasing fruit and vegetable dose appeared to be incrementally greater, the differences were not statistically significant. The absence of dose-response in our review may be explained by ceiling effects, where plasma biomarker concentrations reach a peak and do not increase further in response to higher fruit and vegetable intakes, because excess levels are stored in body tissue or excreted. In the included trials, the difference in fruit and vegetable dose was typically 5-6 portions/d, equivalent in one trial to $194 \mathrm{mg}$ of vitamin $\mathrm{C}$ and $4 \mathrm{mg} / \mathrm{d}$ of $\beta$-carotene ${ }^{(29)}$. Vitamin $C$ saturation can occur at intakes as low as $30-60 \mathrm{mg} / \mathrm{d}^{(40)}$, whereas for $\beta$-carotene doses up to $45 \mathrm{mg} / \mathrm{d}$ are within a physiologically responsive range ${ }^{(41)}$. Ceiling effects may affect vitamin $\mathrm{C}$ but may have less impact on the plasma response of $\beta$-carotene and other carotenoids that have a wider physiologically responsive range. However, our sub-group analyses found no evidence of differences in the pooled effects by baseline fruit and vegetable intake or baseline biomarkers, even for vitamin $\mathrm{C}$ concentrations, indicating that ceiling effects were unlikely to be affecting dose-responses at the tested levels of intake.

Alternatively, trial integrity may have had a role masking a dose-response curve. Adherence to the intervention might be anticipated to be lower for people in groups allocated to higher doses of fruits and vegetables - for example, it is harder to comply with eating 8-9 portions/d than 4 portions/d - and differential compliance by dose may explain the lack of observed dose-response. Shorter (0-3 weeks) compared with longer ( $\geq 4$ weeks) interventions had larger effects, which may be explained by reduced compliance in longer trials owing to intervention fatigue. The half-life of some biomarkers is relatively short, with plasma biomarker concentrations reducing to baseline over 2-3 weeks ${ }^{(41)}$. However, in this review, shorter trials were also more likely to have supervised meals. Five of eight studies of $0-3$ weeks duration (63\%) $v$. three of eleven (27\%) trials of $4+$ weeks duration involved supervised meals. We found that trials with supervised meals had larger pooled effects compared with trials without supervision, likely reflecting better intervention adherence and more accurately representing the intervention-biomarker relationship.

The presence of supervised feeding in trials explained only between $12 \%$ (for $\alpha$-carotene) and $38 \%$ (for lutein) of the between-trial heterogeneity, suggesting that other individual and trial-level factors also influence the observed biomarkerfruit and vegetable intake relationship. Individual-level factors such as age, sex and BMI, the efficiency of absorption and excretion, differences in smoking, alcohol, dietary and exercise habits and variation in the presence of underlying disease/metabolic disorders are suggested influences on the relationship between fruit and vegetable intake and biomarker status $^{(10,41,42)}$. Several of these moderating factors were explored in the sub-group analyses. Health status was identified as a source of heterogeneity; trials that recruited participants who were overweight, hypertensive or at high risk of CVD had lower pooled effect sizes than trials of healthy participants. Factors related to CVD, such as chronic low-grade inflammation, can affect the absorption, metabolism and storage of biomarkers in the body ${ }^{(10)}$, which may explain the reduced effect of interventions in populations with disease/metabolic disturbances. One key trial-level difference not captured fully in our sub-group analyses was the variation in the types of fruits and vegetables provided to participants. Diets with fruits and vegetables that were richer in vitamin $C$ and carotenoids may have shown a stronger relationship with biomarker levels. However, although the type of fruits and vegetables provided was reported in eleven out of nineteen studies, the amount of each type was not consistently described. Without information on both the type and amount of specific fruits and vegetables, it was not possible to accurately estimate the vitamin $\mathrm{C}$ or carotenoid content of diets. We included studies changing more than one type of fruit or vegetable in order to represent 'general' changes in intake, but it is possible that the micronutrient composition of the fruits and vegetables provided could further explain some of the heterogeneity in biomarker responses between studies.

According to the GRADE assessment, the evidence was of low quality; therefore, 'Further research is very likely to have an important impact on our confidence in the estimate of effect and any estimate of effect is very uncertain, ${ }^{,(20)}$. The interpretation of results in this review is limited by the high level of heterogeneity observed between trials, which could not be fully explained in the sub-group analyses. In assessing fruit and vegetable intake not only is there likely to be large betweenpopulation variation but there is also likely to be large variation in the biomarker response of individuals ${ }^{(41-43)}$. The evidence from this meta-analysis does not provide support for the use of biomarkers to estimate absolute levels of fruit and vegetable intake because of a lack of dose-response effect. It also does not provide support for estimating changes in fruit and 
vegetable intake in individuals because only group-level differences were quantified in the trials. Further studies of the determinants of within and between individual variation in vitamin $\mathrm{C}$ and carotenoid levels in large-scale studies with biomarkers measured at multiple time points will help understand the relative importance of changes in fruit and vegetable consumption for changes in biomarker concentrations.

Strengths of the present systematic review include the identification of nine trials additional to the previous review, thus allowing an in-depth exploration of between-trial heterogeneity and a comparative analysis restricted to nine trials with a common set of biomarkers measured (five were newly identified by our update to the review). However, some uncertainty remains regarding the comparative utility of different biomarkers. Although vitamin $\mathrm{C}$ had the greatest response, it was not significantly greater from the response of other biomarkers. Therefore, no particular biomarker can be recommended to be superior to the others based on our results, thus selection may be based on study needs. The review included only RCT that directly observed or provided fruits and vegetables. This restriction reduced the number of included trials compared with previous reviews ${ }^{(14)}$, but is considered a strength because observed effects are less confounded by potential exposure misclassification related to low compliance or other dietary changes associated with dietary interventions.

The present systematic review and meta-analysis confirm that vitamin $\mathrm{C}$ and carotenoids (except lycopene) are responsive to changes in general fruit and vegetable intake at a group level. However, the evidence was of low quality, there was no clear evidence of dose-response or that any single biomarker was more responsive. Further work is required to understand the determinants of biomarker variation among individuals.

\section{Acknowledgements}

The authors thank the authors of the original studies included in this review who provided extra data on request. The authors thank Ann Fry-Smith for assistance in translating the search term.

The work in this article was supported in part by a grant from the European Union (EPIC-CVD, FP7, grant no. 2793233-2). The European Union had no role in the design, analysis or writing of this article.

L. J. formulated the research question; L. J., A. B., C. M., M. S. and M. P. designed the study; M. P., M. S. and L. J. carried out the research. L. J. analysed the data. M. P. and L. J. wrote the article. A. B., M. S. and C. M. revised the paper critically for important intellectual content, all the authors approved the final manuscript.

All the authors have no potential conflicts of interest.

\section{Supplementary material}

For supplementary material/s referred to in this article, please visit http://dx.doi.org/doi:10.1017/S0007114515003165

\section{References}

1. Wang X, Ouyang Y, Liu J, et al. (2014) Fruit and vegetable consumption and mortality from all causes, cardiovascular disease, and cancer: systematic review and dose-response meta-analysis of prospective cohort studies. BMJ 349, g4990.

2. Aune D, Chan DS, Vieira AR, et al. (2012) Dietary compared with blood concentrations of carotenoids and breast cancer risk: a systematic review and meta-analysis of prospective studies. Am J Clin Nutr 96, 356-373.

3. Pereira MA (2013) Diet beverages and the risk of obesity, diabetes, and cardiovascular disease: a review of the evidence. Nutr Rev 71, 433-440.

4. Liu H, Wang XC, Hu GH, et al. (2015) Fruit and vegetable consumption and risk of bladder cancer: an updated meta-analysis of observational studies. Eur J Cancer Prev (epublication ahead of print 30 January 2015).

5. World Health Organization (2004) Fruit and vegetables for health. Report of a joint FAO/WHO workshop. http://www. who.int/dietphysicalactivity/publications/fruit_vegetables_ report.pdf?ua $=1$ (accessed July 2015).

6. Bhattarai N, Prevost AT, Wright AJ, et al. (2013) Effectiveness of interventions to promote healthy diet in primary care: systematic review and meta-analysis of randomised controlled trials. BMC Public Health 13, 1203-1217.

7. Freedman LS, Commins JM, Moler JE, et al. (2014) Pooled results from 5 validation studies of dietary self-report instruments using recovery biomarkers for energy and protein intake. Am J Epidemiol 180, 172-188.

8. Hebert JR, Hurley TG, Peterson KE, et al. (2008) Social desirability trait influences on self-reported dietary measures among diverse participants in a multicenter multiple risk factor trial. $J$ Nutr 138, 226S-234S.

9. Miller T, Abdel-Maksoud M, Crane L, et al. (2008) Effects of social approval bias on self-reported fruit and vegetable consumption: a randomized controlled trial. Nutr J 7, 18-25.

10. Jenab M, Slimani N, Bictash M, et al. (2009) Biomarkers in nutritional epidemiology: applications, needs and new horizons. Hum Genet 125, 507-525.

11. Al-Delaimy WK, Slimani N, Ferrari P, et al. (2005) Plasma carotenoids as biomarkers of intake of fruits and vegetables: ecological-level correlations in the European Prospective Investigation into Cancer and Nutrition (EPIC). Eur J Clin Nutr 59, 1397-1408.

12. Andersen LF, Veierod MB, Johansson L, et al. (2005) Evaluation of three dietary assessment methods and serum biomarkers as measures of fruit and vegetable intake, using the method of triads. BrJ Nutr 93, 519-527.

13. Dehghan M, Akhtar-Danesh N, McMillan CR, et al. (2007) Is plasma vitamin $\mathrm{C}$ an appropriate biomarker of vitamin $\mathrm{C}$ intake? A systematic review and meta-analysis. Nutr J 6, 41-53.

14. Baldrick FR, Woodside JV, Elborn JS, et al. (2011) Biomarkers of fruit and vegetable intake in human intervention studies: a systematic review. Crit Rev Food Sci Nutr 51, 795-815.

15. Rantala M, Silaste ML, Tuominen A, et al. (2002) Dietary modifications and gene polymorphisms alter serum paraoxonase activity in healthy women. J Nutr 132, 3012-3017.

16. Crane TE, Kubota C, West JL, et al. (2011) Increasing the vegetable intake dose is associated with a rise in plasma carotenoids without modifying oxidative stress or inflammation in overweight or obese postmenopausal women. $J$ Nutr 141, 1827-1833.

17. Dragsted LO, Pedersen A, Hermetter A, et al. (2004) The 6-a-day study: effects of fruit and vegetables on markers of oxidative stress and antioxidative defense in healthy nonsmokers. Am J Clin Nutr 6, 1060-1072. 
18. Berry SE, Mulla UZ, Chowienczyk PJ, et al. (2010) Increased potassium intake from fruit and vegetables or supplements does not lower blood pressure or improve vascular function in UK men and women with early hypertension: a randomised controlled trial. Br J Nutr 104, 1839-1847.

19. Higgins JP, Altman DG, Gotzsche PC, et al. (2011) The Cochrane Collaboration's tool for assessing risk of bias in randomised trials. BMJ 343, d5928.

20. Guyatt GH, Oxman AD, Schunemann HJ, et al. (2011) GRADE guidelines: a new series of articles in the Journal of Clinical Epidemiology. J Clin Epidemiol 64, 380-382.

21. Abete I, Romaguera D, Vieira AR, et al. (2014) Association between total, processed, red and white meat consumption and all-cause, CVD and IHD mortality: a meta-analysis of cohort studies. Br J Nutr 112, 762-775.

22. NHS-Choices (2013) 5 A Day portion sizes. http://www.nhs.uk/ Livewell/5ADAY/Pages/Portionsizes.aspx (accessed July 2015).

23. Gill CI, Haldar S, Porter S, et al. (2004) The effect of cruciferous and leguminous sprouts on genotoxicity, in vitro and in vivo. Cancer Epidemiol Biomarkers Prev 13, 1199-1205.

24. Thompson HJ, Heimendinger J, Diker A, et al. (2006) Dietary botanical diversity affects the reduction of oxidative biomarkers in women due to high vegetable and fruit intake. J Nutr 136, 2207-2212.

25. Higgins JP, Thompson SG, Deeks JJ, et al. (2003) Measuring inconsistency in meta-analyses. BMJ 327, 557-560.

26. Baldrick FR, Elborn JS, Woodside JV, et al. (2012) Effect of fruit and vegetable intake on oxidative stress and inflammation in COPD: a randomised controlled trial. Eur Resp $J$ 39, 1377-1384.

27. Briviba K, Bub A, Moseneder J, et al. (2008) No differences in DNA damage and antioxidant capacity between intervention groups of healthy, nonsmoking men receiving 2, 5 , or 8 servings/day of vegetables and fruit. Nutr Cancer 60, 164-170.

28. Broekmans WM, Klopping-Ketelaars IA, Schuurman CR, et al. (2000) Fruits and vegetables increase plasma carotenoids and vitamins and decrease homocysteine in humans. J Nutr $\mathbf{1 3 0}$, $1578-1583$.

29. Chong MF, George TW, Alimbetov D, et al. (2013) Impact of the quantity and flavonoid content of fruits and vegetables on markers of intake in adults with an increased risk of cardiovascular disease: the FLAVURS trial. Eur J Nutr 52, 361-378.

30. McCall DO, McGartland CP, McKinley MC, et al. (2009) Dietary intake of fruits and vegetables improves microvascular function in hypertensive subjects in a dose-dependent manner. Circulation 119, 2153-2160.
31. Neville CE, Young IS, Gilchrist SECM, et al. (2013) Effect of increased fruit and vegetable consumption on physical function and muscle strength in older adults. Age (Dordr) 35 , 2409-2422.

32. van het Hof KH, Tijburg LBM, Pietrzik K, et al. (1999) Influence of feeding different vegetables on plasma levels of carotenoids, folate and vitamin C. Effect of disruption of the vegetable matrix. BrJ Nutr 82, 203-212.

33. Wallace IR, McEvoy CT, Hunter SJ, et al. (2013) Doseresponse effect of fruit and vegetables on insulin resistance in people at high risk of cardiovascular disease: a randomized controlled trial. Diabetes Care 36, 3888-3896.

34. Brevik A, Andersen LF, Karlsen A, et al. (2004) Six carotenoids in plasma used to assess recommended intake of fruits and vegetables in a controlled feeding study. Eur J Clin Nutr $\mathbf{5 8}$, 1166-1173.

35. Moller P, Vogel U, Pedersen A, et al. (2003) No effect of 600 grams fruit and vegetables per day on oxidative DNA damage and repair in healthy nonsmokers. Cancer Epidemiol Biomarkers Prev 12, 1016-1022.

36. Thompson HJ, Heimendinger J, Sedlacek S, et al. (2005) 8-Isoprostane F2alpha excretion is reduced in women by increased vegetable and fruit intake. Am J Clin Nutr 82, $768-776$

37. Thompson HJ, Heimendinger J, Gillette C, et al. (2005) In vivo investigation of changes in biomarkers of oxidative stress induced by plant food rich diets. I Agric Food Chem $\mathbf{5 3}$, 6126-6132.

38. Martini MC, Campbell DR, Gross MD, et al. (1995) Plasma carotenoids as biomarkers of vegetable intake: the University of Minnesota Cancer Prevention Research Unit Feeding Studies. Cancer Epidemiol Biomarkers Prev 4, 491-496.

39. Howe JA, Valentine AR, Hull AK, et al. (2009) 13C natural abundance in serum retinol acts as a biomarker for increases in dietary provitamin A. Exp Biol Med (Maywood) 234, $140-147$.

40. Levine M, Dhariwal KR, Welch RW, et al. (1995) Determination of optimal vitamin $\mathrm{C}$ requirements in humans. Am J Clin Nutr 62, 1347S-1356S.

41. Dimitrov NV, Meyer C, Ullrey DE, et al. (1988) Bioavailability of beta-carotene in humans. Am J Clin Nutr 48, 298-304.

42. Giovannucci E (2013) Nutrient biomarkers are not always simple markers of nutrient intake. Am J Clin Nutr 97, 657-659.

43. van Het Hof KH, West CE, Weststrate JA, et al. (2000) Dietary factors that affect the bioavailability of carotenoids. $J$ Nutr 130, 503-506. 